\title{
Design of a Resistively Heated Thermal Hydraulic Simulator for Nuclear Rocket Reactor Cores
}

\author{
Ron J. Litchford ${ }^{1}$, John P. Foote ${ }^{2}$, Narayanan Ramachandran ${ }^{3}$, and Ten-See Wang ${ }^{4}$ \\ NASA Marshall Space Flight Center \\ Samim Anghaie ${ }^{5}$ \\ University of Florida, Innovative Nuclear Space Power and Propulsion Institute (INSPI)
}

\begin{abstract}
A preliminary design study is presented for a non-nuclear test facility which uses ohmic heating to replicate the thermal hydraulic characteristics of solid core nuclear reactor fuel element passages. The basis for this testing capability is a recently commissioned nuclear thermal rocket environments simulator, which uses a high-power, multi-gas, wall-stabilized constricted arc-heater to produce high-temperature pressurized hydrogen flows representative of reactor core environments, excepting radiation effects. Initially, the baseline test fixture for this non-nuclear environments simulator was configured for long duration hot hydrogen exposure of small cylindrical material specimens as a low cost means of evaluating material compatibility. It became evident, however, that additional functionality enhancements were needed to permit a critical examination of thermal hydraulic effects in fuel element passages. Thus, a design configuration was conceived whereby a short tubular material specimen, representing a fuel element passage segment, is surrounded by a backside resistive tungsten heater element and mounted within a selfcontained module that inserts directly into the baseline test fixture assembly. With this configuration, it becomes possible to create an inward directed radial thermal gradient within the tubular material specimen such that the wall-to-gas heat flux characteristics of a typical fuel element passage are effectively simulated. The results of a preliminary engineering study for this innovative concept are fully summarized, including high-fidelity multi-physics thermal hydraulic simulations and detailed design features.
\end{abstract}

\section{Introduction}

$\mathrm{S}$ olid core nuclear thermal rocket (NTR) engines which directly heat hydrogen propellant in high temperature fuel elements can, in principle, serve as highly efficient space thrusters with envisioned specific impulse performance ranging as high as $900-1,000$ secs. The attractiveness of this performance potential could not be ignored during the heydays of the cold war space race, and several large technology development programs were instituted at US National Laboratories and former Soviet Institutes in the USSR for the purpose of realizing a practical nuclear thermal flight engine. These programs were well funded, well led, and well directed, and great technological strides were indeed made toward this goal before the perceived need faded away. ${ }^{[1-6]}$. It should be noted, however, that these efforts also revealed a number of performance and life limiting mechanisms for graphiteand carbide-based fuels that were never fully resolved. Of principle significance was the observation that many interrelated and competing physical processes were acting in concert to degrade structural integrity and accelerate fuel mass loss. ${ }^{[7-10]}$ Chief among these were the formation of liquid, vaporization (sublimation), creep of material defects, local corrosion, and structural degradation.

\footnotetext{
${ }^{1}$ Project Principal Investigator, Thermal/Combustion Devices, Propulsion System Dept., Associate Fellow AIAA.

${ }^{2}$ Project Engineer (Jacobs/Sverdrup), Thermal/Combustion Devices, Propulsion Systems Dept.

${ }^{3}$ Project Analyst (Jacobs/Sverdrup), Thermal Analysis, Propulsion Systems Dept.

${ }^{4}$ Technical Assistant, Thermal/Combustion Analysis, Propulsion Systems Dept., Senior Member AIAA.

${ }^{5}$ Project Consultant, Professor, Department of Nuclear and Radiological Engineering; and Director, Innovative Nuclear Space Power and Propulsion Institute (INSPI).
} 
All of these highly complex physical phenomena give rise to challenging reactor core design problems, and straightforward engineering solutions are generally difficult to identify and implement. The critical technical obstacle to a practically useful NTR engine, therefore, continues to be the development of innovative hightemperature reactor core materials that can endure 2,500-3,000 $\mathrm{K}$ hydrogen environments with minimal corrosion under pressures in excess of $3 \mathrm{MPa}$ while also possessing sufficient strength and resilience to resist breakage from intense vibration loads and thermally induced stresses. Moreover, the need to optimize propulsion system power density invariably leads to extremely high radiation flux operating conditions, which can create material point defects as an onset to cracking thereby accelerating corrosion rates.

In view of the limited knowledge on phase relationships and stability of these carbon systems as well as the significant advances in high temperature material formulations and processing over the intervening years, it is suggested that reactor fuel technology be revisited and thoroughly re-examined prior to embarking on a modern NTR engine development path. Promising contemporary approaches for realizing additional extensions in operating temperature and lifetime, for instance, include utilization of modern coating techniques and advanced hightemperature materials such as bi/tri-carbides of the $\mathrm{U}-\mathrm{Zr}-\mathrm{X}$ type, carbon-nitrides of $\mathrm{U}$ and $\mathrm{Zr}$, and CERMETs based on a $\mathrm{W}$ matrix.

Unfortunately, it would be extremely difficult to justify the high costs of in-core nuclear fuel development testing in the absence of a full blown NTR engine development program. As a counterpoint, it should also be pointed out that the establishment of a modern engine development program would most likely be so hamstrung by cost and schedule constraints that it would be impossible to tolerate incorporation of any in-depth technology development activities. This Catch 22 situation has been a consistent plague and serious constraint on nuclear propulsion technology innovation for decades, and, for these reasons, non-nuclear testing methods should be looked to as a potential means of reducing the complexity and costs of upfront fuel development R\&D. Admittedly, skeptical technical specialists tend to view non-nuclear fuels testing as being deficient in several respects, mainly because it is unable to properly account for radiation effects which are known to have a significant influence on corrosion in NTR reactor core environments. ${ }^{[10,11]}$ Nevertheless, it is our view that, as an interim step, considerable understanding and development progress could be made with these techniques, without which advances in hightemperature fuel technology may prove unrealizable.

Recently, an NTR environments simulator was developed and placed into service at NASA Marshall Space Flight Center (MSFC), which uses a high-power, multi-gas, wall-stabilized constricted arc-heater to produce hightemperature pressurized hydrogen flows representative of reactor core environments, excepting radiation effects. ${ }^{[2]}$ Initially, the baseline test fixture for this non-nuclear environments simulator was configured for long duration hot hydrogen exposure of small cylindrical material specimens as a low cost means of evaluating material compatibility, as illustrated in Fig. 1. It became evident, however, that additional functionality enhancements were needed to

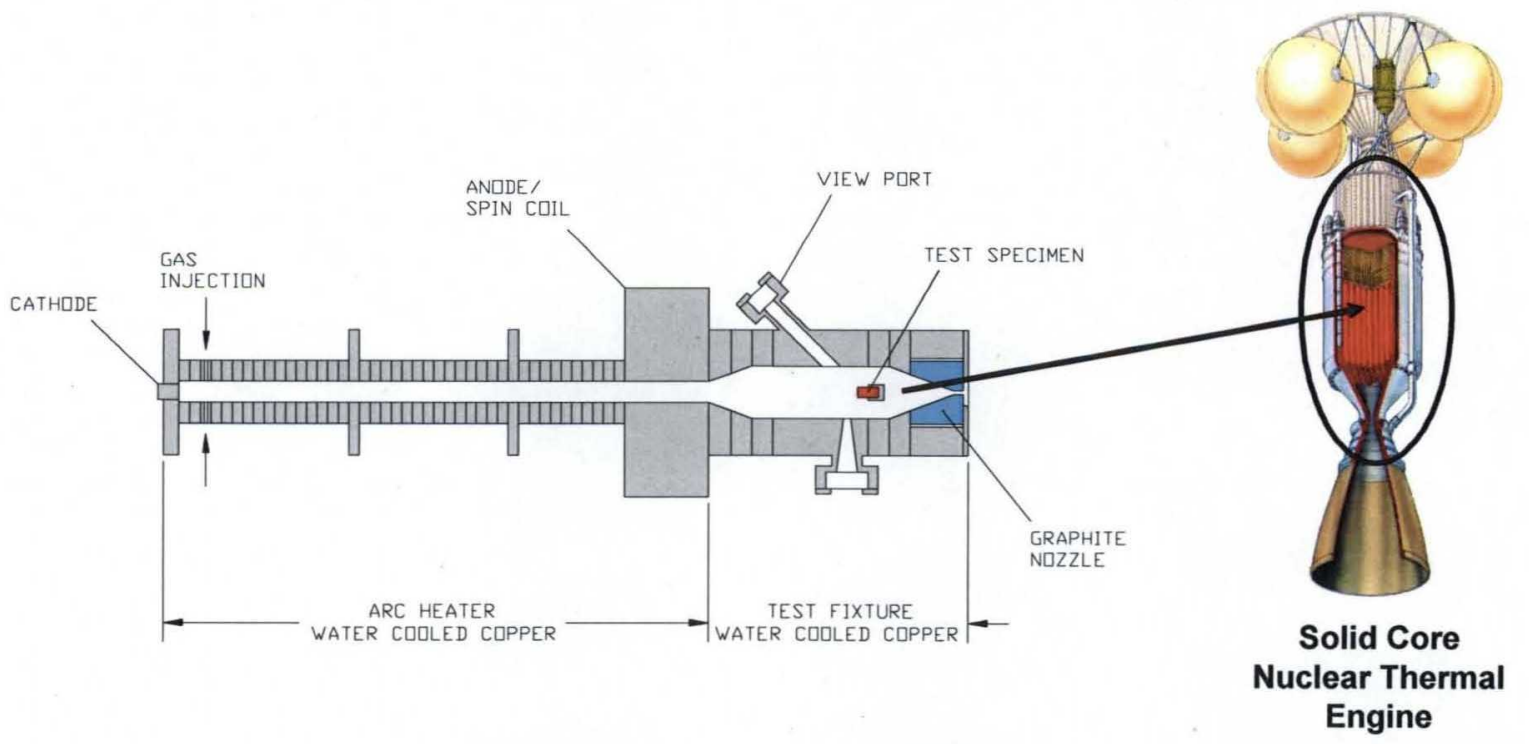

Figure 1. Baseline configuration of arc-heater driven nuclear thermal rocket environment simulator for the generation of hyper-thermal convective conditions traceable to reactor core environments. 
permit a critical examination of thermal hydraulic effects in fuel element passages. Thus, a design configuration was conceived whereby a short tubular material specimen, representing a fuel element passage segment, is surrounded by a backside resistive tungsten heater element and mounted within a self-contained module that inserts directly into the baseline test fixture assembly.

In the envisioned system, hydrogen gas is pre-heated in the high power arc-heater and forced to flow through the central passage of the tubular specimen, where additional heat can be electrically generated and transferred to the backside of the specimen. With this configuration, it becomes possible to create an inward directed radial thermal gradient within the tubular material specimen such that the wall-to-gas heat flux characteristics of a typical fuel element passage are effectively simulated. Thus, by precisely tuning hydrogen gas temperature at the module entrance and precisely depositing ohmic power in the specimen, this arrangement can accurately replicate thermalhydraulic conditions at any location in a high-performance solid core nuclear thermal rocket reactor. This would be a powerful and unique capability without contemporary comparison, and would enable low-cost, rapid-turnaround testing of advanced high-temperature nuclear fuels in a non-irradiated environment. It would also permit direct experimental access to hydrogen corrosion phenomena at extreme temperature under flowing conditions, with unprecedented real-time measurement diagnostics, and provide valuable data for corrosion modeling development.

\section{Technical Approach}

This article presents the results of a preliminary engineering study for the suggested thermal hydraulic simulation module, including high-fidelity multi-physics thermal hydraulic simulations and detailed design features. In approaching this challenging design problem, it was first necessary to establish a rudimentary conceptual layout using the configuration, dimensions, and specifications of the existing baseline test fixture as rigorous constraints on the available design space. Then, comprehensive thermal-fluid simulations were performed, including conjugate heat transfer, which served to confirm system performance characteristics. Various mechanical design refinements were considered, and thermal/stress constraints were satisfied to insure safe operation. Using the general conceptual layout as a guidepost, detailed engineering solutions were developed for the mechanical/flow-path interfaces, water coolant interfaces, electrical power supply/components/connections, heater element configuration, and diagnostics access. Finally, design drawings were developed, and specifications were set for special test equipment and components. Potential vendors for these procurements were identified and preliminary cost estimates were developed to support a future follow-on acquisition and fabrication phase.

\section{Baseline Facility}

\section{A. Description}

The facility arc-heater is energized by a saturable reactor DC power supply which can sustain a continuous operating power of $0.75-\mathrm{MW}_{\mathrm{e}}$ on an indefinite basis and can deliver an intermittent power burst of $1.5-\mathrm{MW}_{\mathrm{e}}$ for 5 to 10 minutes. A detailed description of this device and its operational attributes were thoroughly presented along with in a companion paper to which the interested reader should refer. ${ }^{[12]}$ An analysis of arc-heater performance characteristics subject to facility operating constraints indicated that reactor-like environments could be sustained indefinitely over a temperature range of $2,500-3,500 \mathrm{~K}$ while flowing $7-10 \mathrm{~g} / \mathrm{s}$ of hydrogen. Because this result was relatively insensitive to pressure, the maximum chamber pressure for testing was governed by the flow supply system ratings of the facility infrastructure and structural limits of the arc-heater and test fixture attachments.

Based on these constraints, baseline test fixture design parameters were specified, as summarized in Table 1, and a modular water-cooled test fixture was designed and developed in which small rod-shaped material specimens could be securely mounted and exposed to the arc-heated hydrogen flow through direct attachment to the arc-heater exhaust flange. This apparatus also included optical access features for real-time pyrometer measurement of specimen surface temperature and non-intrusive laser-based measurement of the hydrogen thermodynamic state.

The final layout for the baseline test fixture is shown in Fig. 2. Note that design modularity is achieved by configuring the test fixture as a stack of water-cooled copper segments which attach directly to the arc-heater anode section. The mid-section contains features for insertion of a tungsten spider to hold small material specimens (1-inch long, $1 / 2$-inch diameter rods) and has

Table 1. Test Fixture Design Specifications.

\begin{tabular}{ll}
\hline \hline$T_{\max }$ & $3,500 \mathrm{~K}$ \\
$p_{\max }$ & $35 \mathrm{~atm}$ \\
$\dot{m}_{\text {hydrogen }}$ & $5-10 \mathrm{~g} / \mathrm{s}$ \\
$d_{\text {bore } .}$ & 2.5 inches \\
$d_{\text {specimen }}$ & 0.5 inches \\
$l_{\text {specimen }}$ & 1.0 inches \\
\hline \hline
\end{tabular}




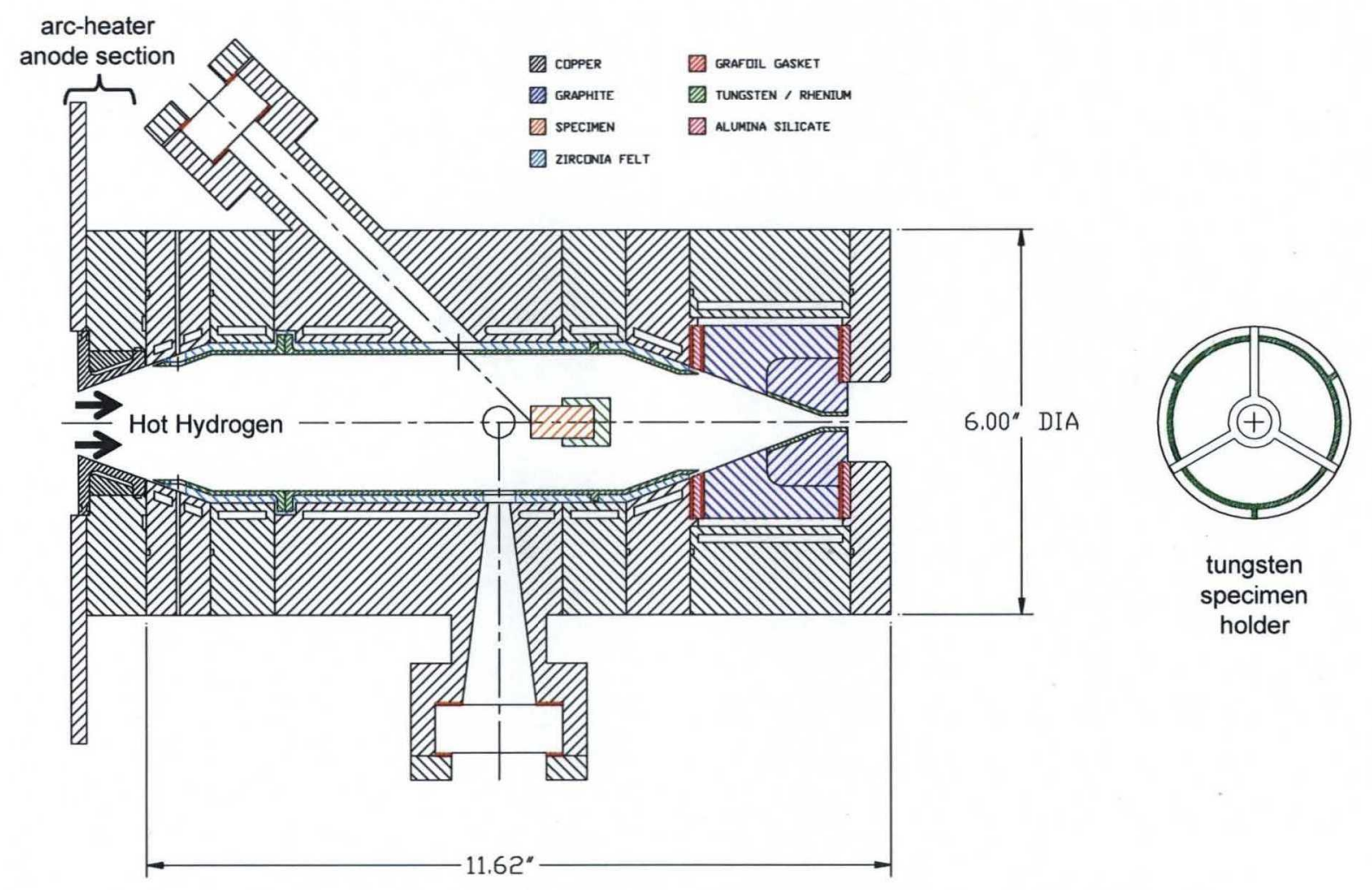

Figure 2. Baseline test fixture layout drawing for the hot hydrogen nuclear thermal environments simulator.

four windowed ports for optical access. One port is canted to allow pyrometer measurements of specimen surface temperature during exposure. Two diametrically opposed ports allow for focusing of a laser excitation beam at a location just upstream of the specimen nose, and a fourth port is rotated $90^{\circ}$ out of this plane for capture of Raman scattering from diatomic hydrogen, which can be used to infer the rotational temperature and number density of molecular hydrogen. The hot gas exiting the specimen exposure section is directed through additional segments forming a converging flowpath and is throttled through a graphite nozzle with a tungsten throat insert.

In order to minimize radiation losses through the highly transparent hydrogen and to keep cold wall heat flux below the commonly accepted $500 \mathrm{~W} / \mathrm{cm}^{2}$ threshold, a 0.060 -inch thick W/5\%Re heat shield was employed in the design with a 0.125 -inch gap between the shield and the copper wall, as shown in the layout drawing of Fig. 2. A ring-shaped dam was also incorporated on the outer surface of the shield to prevent seepage of hot hydrogen flow through the backside gap. Further detailed information on test fixture design and fabrication may be found in a companion paper. ${ }^{[12]}$

\section{B. Performance}

Baseline arc-heater performance capability was established by attaching a sacrificial graphite nozzle assembly directly to the arc-heater anode flange. The sacrificial nozzle was machined from high-density graphite with a throat diameter matching the final test fixture design specifications so that the proper flow rate and chamber pressure would be produced at the specified mass flow rate and input power settings. This approach provided a reliable method for accurate performance characterization while test fixture hardware was in fabrication. Performance mapping tests were then conducted to empirically correlate operational parameters with the controllable input settings. In this case, the two controllable input parameters were the applied arc current and the mass flow rate whereas the applied voltage of the power supply was uniquely determined by the arc plasma impedance for the applied current setting. A detailed account of these performance testing results is provided in a companion paper. ${ }^{[12]}$

More concisely, the results may be reduced to the arc-heater performance map shown in Fig. 3 where the inferred chamber temperature has been correlated as a function of applied current and hydrogen mass flow rate. Symbols indicate data points used to construct the map, and the chamber temperature at each point was estimated by using the inferred thermal input power to define a pseudo heat of combustion for a NASA CEA based rocket 
calculation. Bounds on the operating range are indicated by solid lines on the map. Within this bounded region, the accessible hydrogen temperature ranges from 2,400 to $3,100 \mathrm{~K}$, as needed to simulate NTR reactor environments. If less stressful start-up and shut-down transients are desired to avoid thermal shocking of material specimens, the temperature may be temporarily forced lower by introducing additional cold gas through four gas injection ports in the first expansion ring of the test fixture.

\section{Ohmic Module Design \& Analysis}

\section{A. Notional Design}

The ohmic thermal hydraulic simulator module is required to be geometrically compatible with the baseline test fixture hardware and must feature proper stability in flowing hydrogen environment under the entire range of operating pressure and temperature. Rod, tube, bar, plate or circular shapes could be used to construct the resistive heater element, but a coil shaped configuration was considered superior since it would be compatible with the test fixture geometry and provide more uniform heating.

At the outset of the project, a variety of materials were to be considered for construction of the resistive heater element. The alternatives included coated high density graphite or glossy carbon material and refractory metals. In a vacuum environment, it is not possible to operate a resistive heating element at temperatures above $2,000 \mathrm{~K}$ due to excessive sublimation of graphite. In high pressure hydrogen $(100-500 \mathrm{psi})$, on the other hand, high density graphite heating elements could be operated at temperatures up to $2,300 \mathrm{~K}$ without significant loss of carbon and hydrocarbon formation. Glossy carbon features lower corrosion and evaporation rates and could be used for operation at temperatures up to $2,500 \mathrm{~K}$. For operation at temperatures above 2,500 $\mathrm{K}$, however, it would be necessary to cost the graphite heating element using transition metal refractory carbides such as tantalum or hafnium carbides. Thus, the design phase of the project included detailed material and chemical compatibility analysis for coated graphite materials. Some exploratory testing was also undertaken to assess the degree of chemical compatibility of glossy carbon and coated graphite with hot hydrogen to a maximum temperature of $3,200 \mathrm{~K}$.

Using the predefined requirements and specifications as a guide, we first arrived at a notional design concept illustrated in Fig. 4. The general idea was to direct the hot hydrogen from the arc-heater to a constant are test section in which tubular or solid cylindrical specimens up to $8 \mathrm{~cm}$ in diameter would be surrounded and heated by a curved slat resistive heating element. An alumina/zirconia insulator shield would be located between the heater element and the water-cooled copper walls of the test section. A thin sheath of tungsten alloy (W-Ni-Cu) would be placed between the heating element and the insulating material to partially reflect radiative heat back toward the material test specimen.

\section{B. Detailed Design}

Further refinement of the design concept was achieved by imposing geometrical constraints associated with the pre-existing test fixture hardware. The objective was to modify the design as a means of maximizing use of existing hardware and minimizing new hardware fabrication requirements. This effort resulted in a convergence of the design with notable simplifications. In particular, the refined design would only require the construction of a single 
new test section module, which would contain the heater element. Other retrofits included a new specimen mount and a new W/Re alloy shield/funnel section, as shown in the final design layout of Fig. 5. Standard size pieces and fittings were used to keep the number of custom components to a minimum. The key advantage of this approach was the fact that only a single stack of the passive heating test fixture hardware need be replaced yielding system simplicity and cost containment without sacrificing testing capability. Moreover, the original optical access segment could be retained to provide line of site pyrometer measurement of specimen surface temperature.

Critical innovative design features were mainly associated with the resistive heater element itself. The major technical issues of concern include materials selection for the heater element and its structural support, electrical power feed-through/connections, and heater performance characteristics. Careful examination of the design problem resulted in two possible material alternatives for the curved slat heater element material. The alternatives being either coated graphite or W/Re alloy construction. As originally envisioned, the heater element would be supported by the tubular specimen using four synthetic diamond wafers, as illustrated in the layout of Fig. 5. Since diamond exhibits excellent thermal conductivity characteristics while acting as a near perfect electrical insulator it was believed to be the prime candidate structural support material. How well synthetic diamond major can endure the corrosive hot hydrogen environment was a major unknown, however. To address some of these material compatibility issues during the early stages of the design, some basic hot hydrogen exposure tests were carried out, as will be described below.

A variety of high density graphite materials and glossy carbon were considered for the design of the resistive heating element module, as illustrated in Fig. 6. Since continuous operation at temperatures above 2,300 K was required, it was decided to examine the viability of graphite coated with transition metal carbides such as niobium, tantalum or hafnium carbides. During the early stages of the project and in conjunction with another ongoing research project, several coating trials were performed on three different types of graphite material in order to down select the type of graphite that could be used for the curved slat heater. Generic graphite, JP1060, and isographite material were explored as possible substrate candidates for subsequent deposition by direct and reactive electron beam physical vapor deposition (EB-PVD). Preliminary trials were conducted with $\mathrm{TaC}$ deposited by reactive ion beam assisted (RIBA) deposition since it has the greatest potential for producing single phase TaC through control of carbon deposited on the substrate. Following these early trials, the down selection to $\mathrm{NbC}$ was made due to the large number of $\mathrm{TaC}$ samples that contained other phases including $\mathrm{Ta}_{2} \mathrm{C}$. Coating trials were then carried out with $\mathrm{NbC}$ on 3.5-inch long cylindrical samples using both direct and reaction EB-PVD. The samples were then hot hydrogen tested in a furnace. The results were mixed with regards to the type of graphite and which method of niobium carbide deposition performed the best due to the geometry of the substrate material. Since the substrates

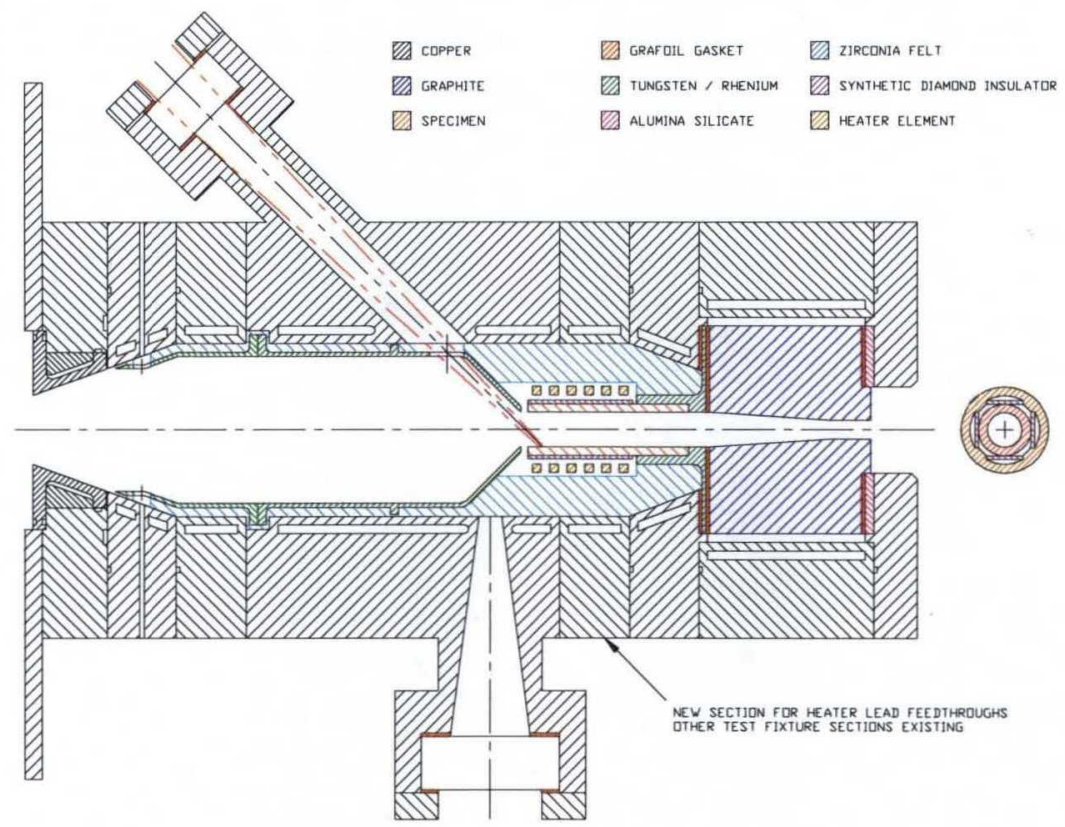

Figure 5. Detailed layout of resistively heated thermal hydraulic simulator module. 


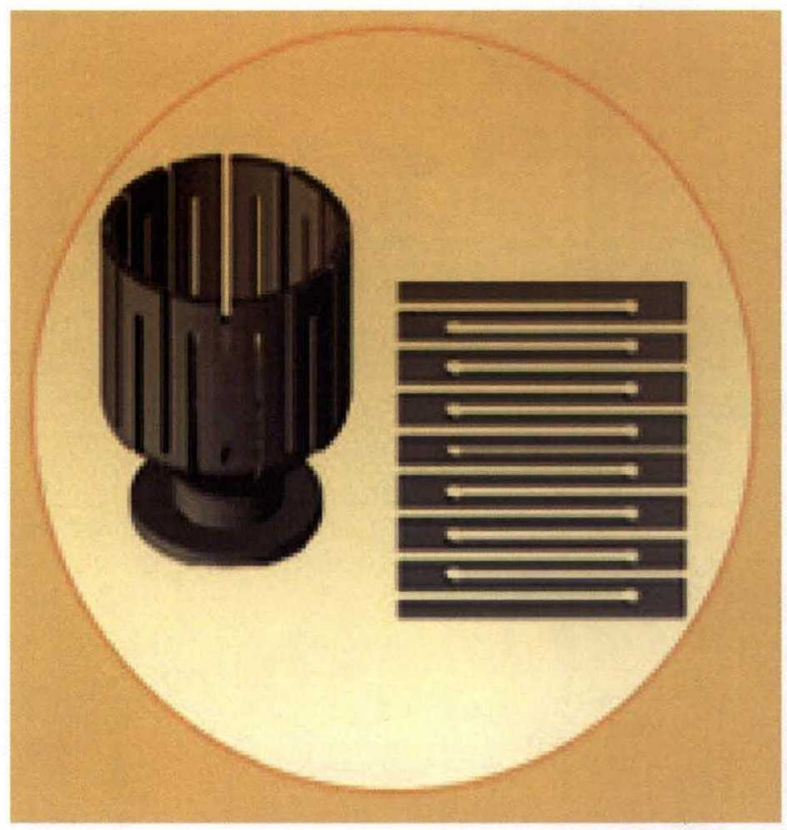

Figure 6. High density graphite curved slat heating element coated with transition metal carbide.

were longer than the hot zone for the initial hot hydrogen testing, increased thermal shock led to premature spallation of the samples. Representative post test images of these spallated specimens are shown in Fig. 7. Due to time limitation on this project, it was decided not to pursue the coated graphite option and the focus was shifted to design of a tungsten base curved slat heating element. Tungsten and its alloys are fully compatible with hydrogen at temperatures up to 3,500 $\mathrm{K}$. The main constraint with tungsten heating element is the fabrication of a single piece heating element with proper dimensions and power leads.

A detailed thermal and mechanical analysis was performed to arrive at design specifications and performance characteristics for the tungsten base resistive heating element module. The electrical resistivity of CVD tungsten in the operational temperature range of the hot hydrogen test facility is within $5-10 \mu \Omega-\mathrm{cm}$. Given the geometrical dimensions of the test section as a constraint, the thickness of the curved slat heating element was adjusted to provide sufficient heating to maintain the test specimen at maximum temperature of $3,500 \mathrm{~K}$. The power requirements for the heating module was determined using detailed CFD and conjugate heat transfer analyses of the entire test section that included typical test specimens and hot hydrogen flow, as well as thermal losses to the boundary. The resulting design of the tungsten curved slat resistive heating element is depicted as a 3D model in Fig. 8.
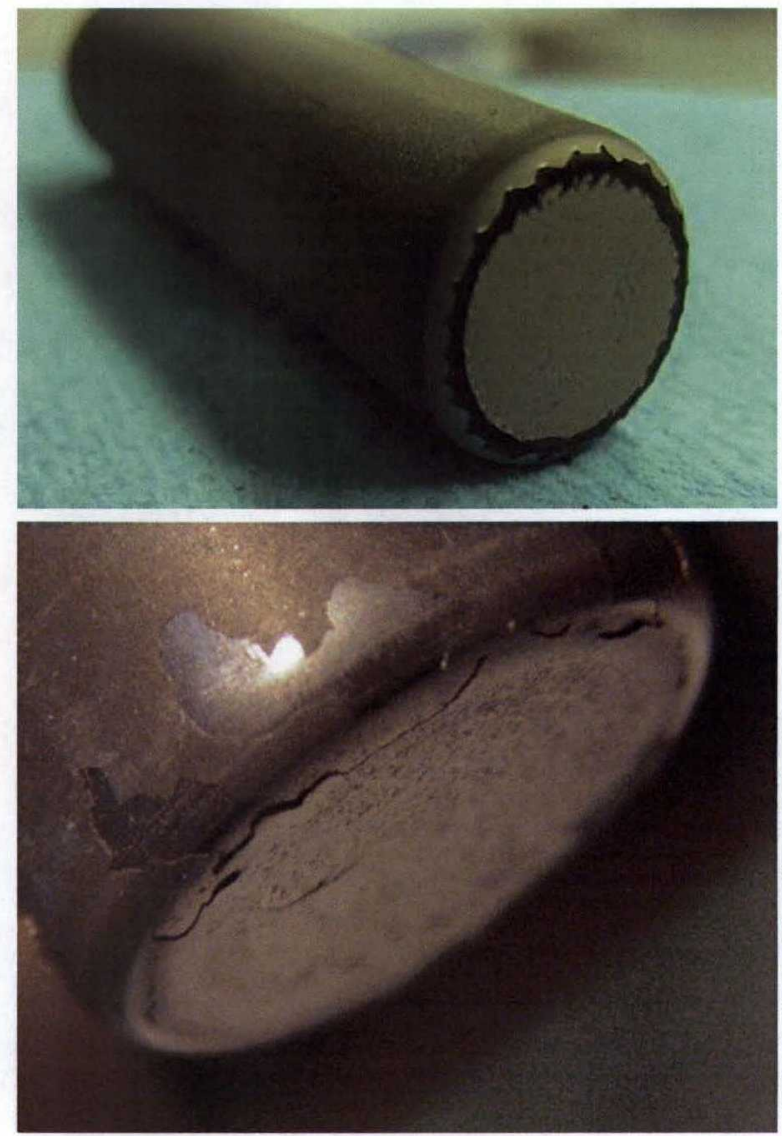

Figure 7. Post-test images of $\mathrm{NbC}$ coated high density graphite specimens following hot hydrogen exposure. Note premature spallation due to thermal shock.

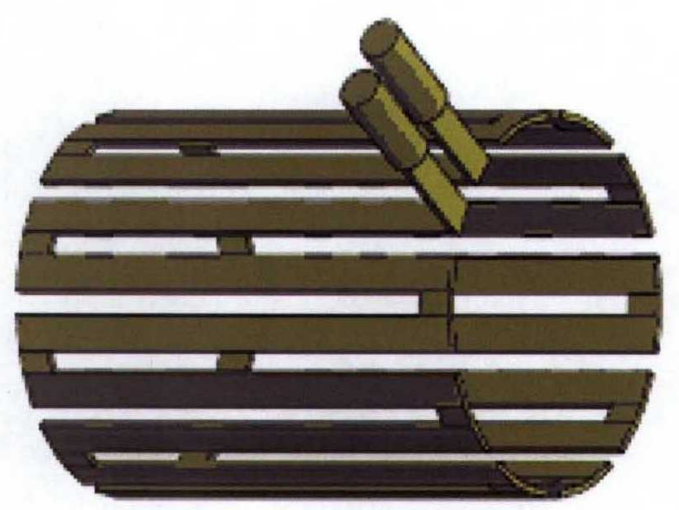

Figure 8. Design of tungsten curved slat resistive heating element. 


\section{Multi-Physics Analysis}

A modified version of FLUENT was combined with a conjugate heat transfer model for thermal-fluid simulation and analysis of the electrically heated test fixture layout shown in Fig. 4. This simulation assumes axial symmetry and accounts for compressible dissociating turbulent hydrogen flow with radiative heat transport. The computational domain for the simulations is provided in Fig. 9. In this particular case, $10 \mathrm{~g} / \mathrm{s}$ of hot hydrogen flow is heated to $2,500 \mathrm{~K}$ in the arc-heater and enters the test fixture at $35 \mathrm{~atm}$ in a uniformly mixed state.

Results from the simulation with a heater temperature set to 3,500 K are summarized in Fig. 10 which show the stream function and temperature distributions in the test fixture. In general, we obtain clean flow structure and good temperature distribution at the entrance to the tubular specimen. Note that the tubular sample is approximately twice the length of the heater in this particular case. The simulation also shows that the heater element performs well and is effective in transferring heat to the backside of the material sample such that the temperature gradient is directed inward through the tube to the hydrogen gas, as evidenced by the growing thermal boundary layer along the inner tube wall. It was found that the specimen temperature could be readily raised and stabilized using the heater element power as a control parameter. The temperature distribution in the specimen and the internal surface temperature along the tube are shown more clearly in the exploded plot of Fig 11.

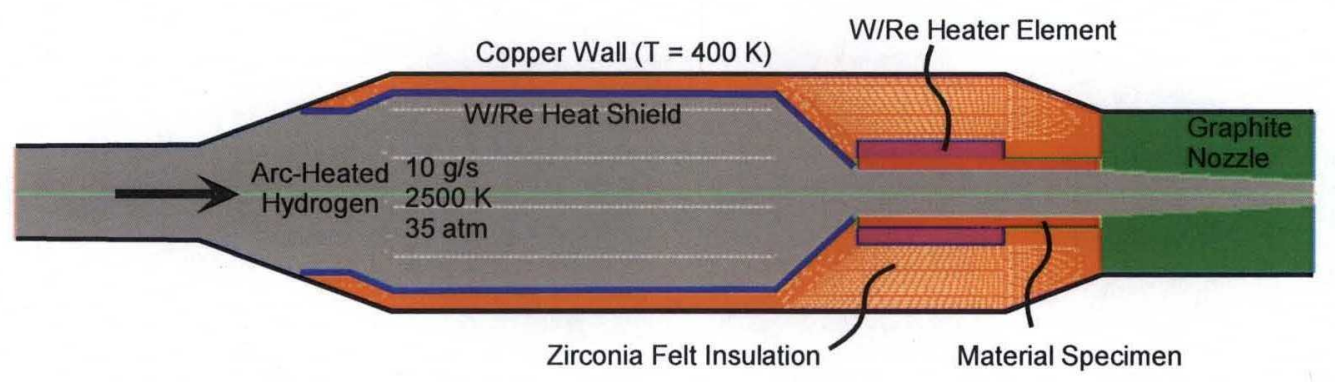

Figure 9. Computational domain for thermal-fluid simulations.

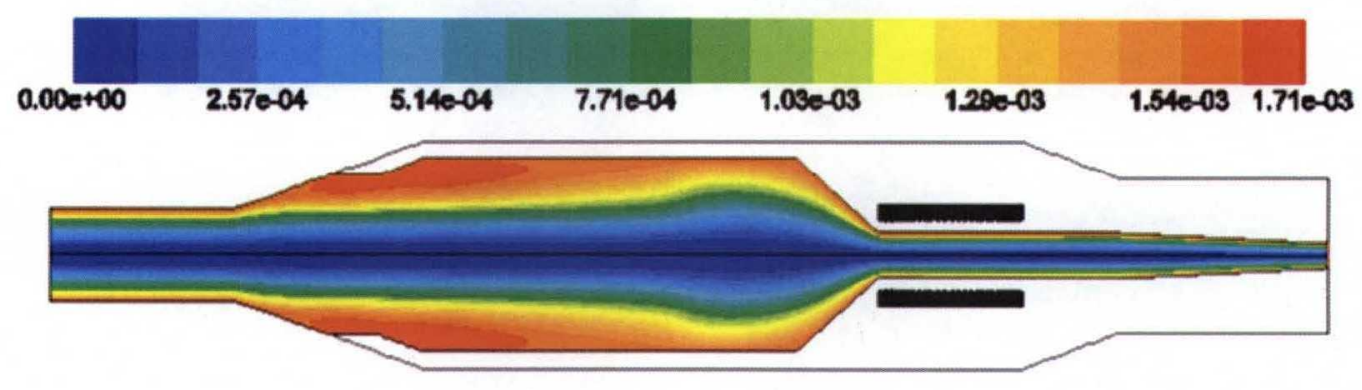

Stream Function $(\mathrm{kg} / \mathrm{s})$

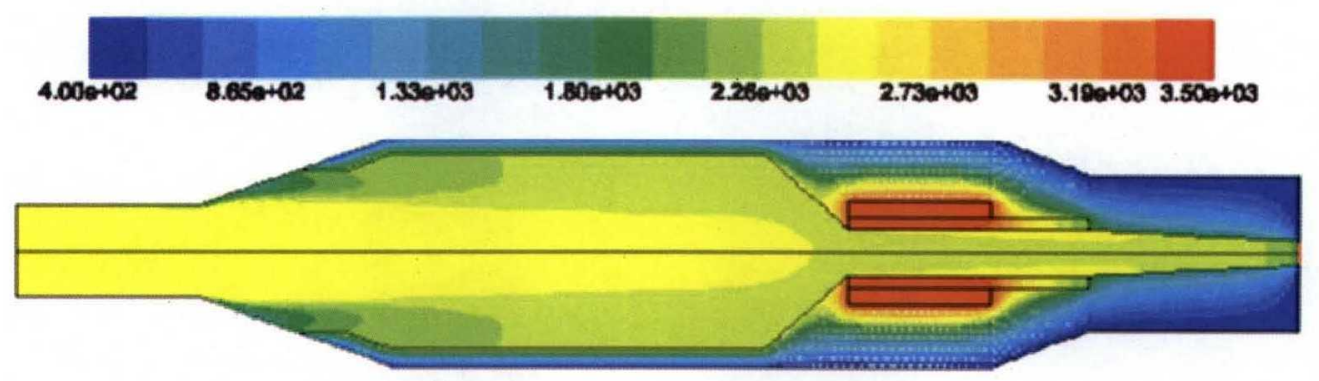

Temperature (K)

Figure 10. Calculated stream function and temperature distributions in test fixture. 

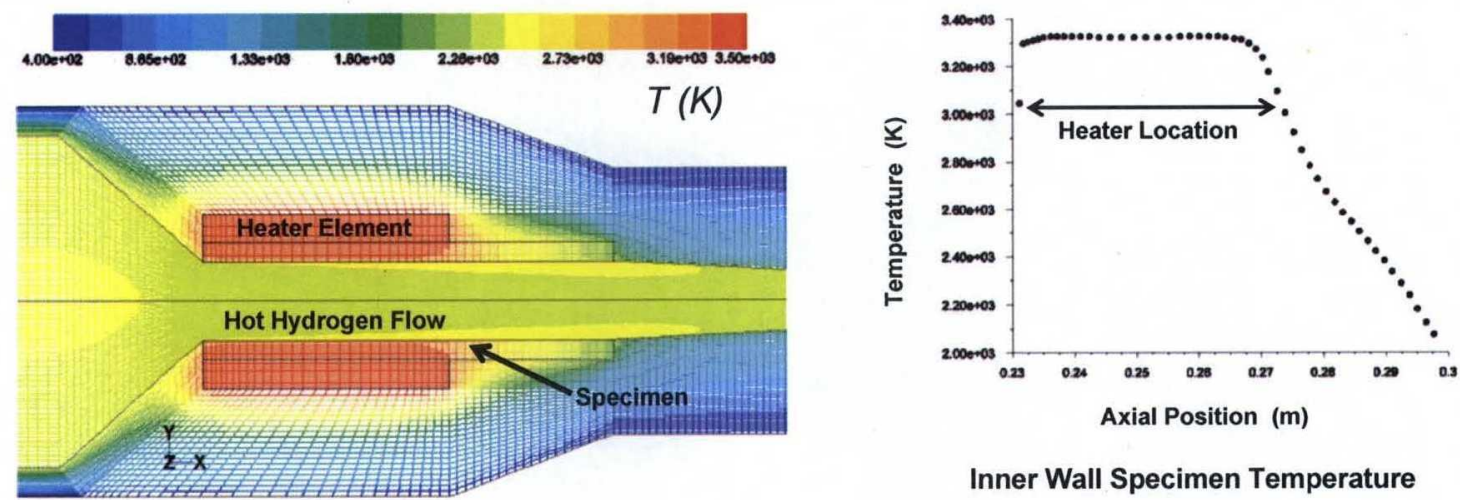

Inner Wall Specimen Temperature

Figure 11. Internal temperature distributions and inner wall surface temperature of specimen.

\section{Synthetic Diamond Hydrogen Compatibility Testing}

The key design issue in any high temperature system is materials. Here, the challenge is made unusually difficult by the corrosive action of hot hydrogen and the need to insulate exposed electrical circuit elements. Of particular concern was the desire to securely mount the resistive heating element without creating a short circuit to ground through the supporting structure. As one possibility, the heating element could simply be suspended by its two electrical leads, which would require a rather intricate feed-through design in order to simultaneously provide high-temperature compatibility, electrical insulation, and adequate structural strength and rigidity. Alternatively, one could consider directly supporting the ohmic heating element on the material specimen if a suitable standoff material was available that would provide both electrical insulation and strength at temperature. At the outset, we were strongly inclined to adapt the latter approach, if a suitable standoff material could be identified.

The material which comes immediately to mind is diamond, since it exhibits excellent thermal conductivity characteristics while acting as a near perfect electrical insulator. The difficulty with natural diamond, of course, is cost and availability in useful structural forms and sizes. Synthetic diamond, on the other hand, is relatively cheap and readily available in large wafers since it is widely used in the manufacture of modern solid state electronic chips. Therefore, our original design layout incorporated four synthetic diamond wafers to structurally support the ohmic heater element on the tubular material specimen, as shown in Fig.5. The significant unknown, however, was whether the synthetic form would have the resilience to survive the severely corrosive hot hydrogen environment.

To answer this critical question, we decided to incorporate a synthetic diamond materials compatibility test into a planned long duration demonstration of the baseline NTR simulator apparatus. To do so, the graphite nozzle section was modified to accommodate the insertion of a synthetic diamond wafer sample as illustrated in Fig. 12. This modification was accomplished by simply splitting the inner graphite shell so that the wafer could be slid into the gap just upstream of the tungsten throat insert.
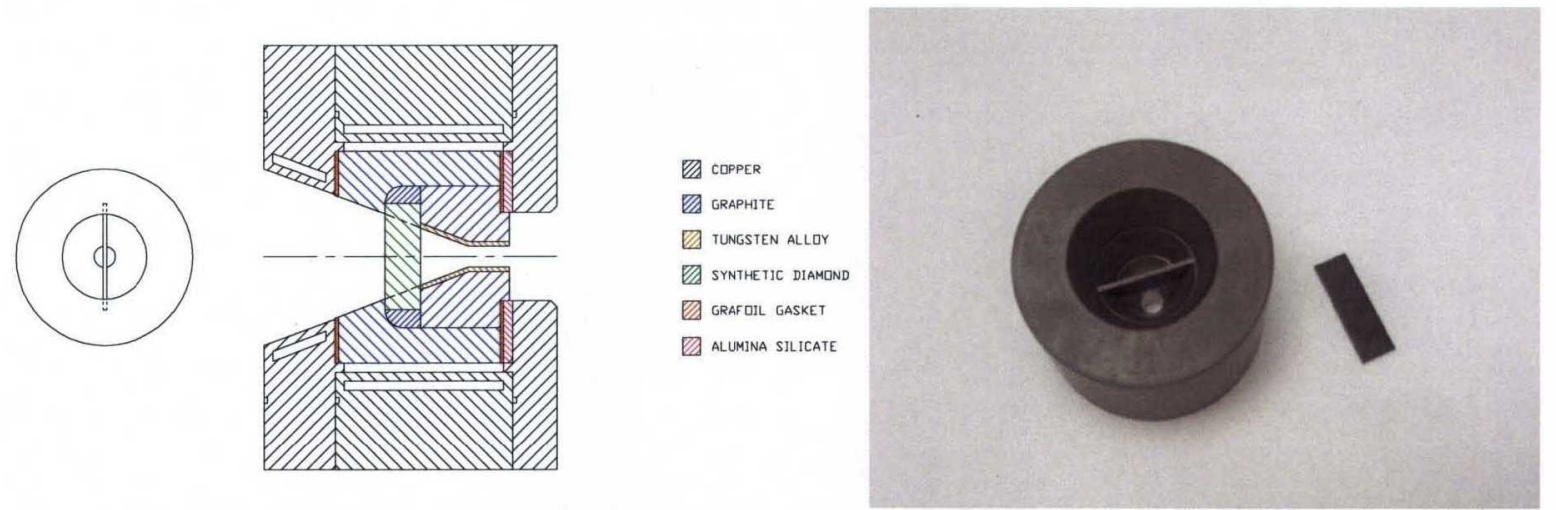

Figure 12. Drawing of baseline graphite nozzle module as modified to accept synthetic diamond wafer specimen and a photograph of the fabricated and assembled components. 
As part of performance verification efforts for the baseline NTR environments simulator, a long duration capability demonstration test was planned in which a W-5\%Re/40\%HfN CERMET material specimen would be exposed to flowing hot hydrogen. Exploiting this opportunity to the fullest, we also installed the modified graphite nozzle module with the synthetic diamond wafer insert prior to the test. The targeted op-point chamber conditions were $10.5 \mathrm{~g} / \mathrm{s}$ hydrogen with an arc current of 235 amps, implying a chamber temperature very near $2,500 \mathrm{~K}$. A complete description of the operating parameters and overall test results are presented in a companion paper. ${ }^{[12]}$ The discussion here is confined to the results of the synthetic diamond material compatibility results.

The total run time for this particular test was slightly in excess of 26 minutes with an inferred gas temperature of $2,516 \mathrm{~K}$, which was consistent with pyrometer measurements of the CERMET specimen temperature. A photograph of the plume during this long duration test is provided in Fig. 13 where the yellow color is indicative of significant carbon loss inside the chamber. Naturally, this carbon could only originate from either the diamond wafer or the exposed graphite surface. Post-test disassembly and inspection revealed that the culprit was indeed the diamond wafer which had been completely ablated away during the test, as shown in Fig. 14. Despite our original hopes for the material, these test results were absolutely conclusive. Synthetic diamond is clearly incompatible with hot hydrogen and the design approach would have to be abandoned.

Thus, the diamond wafer support structures in the original layout will have to be removed as part of a future redesign effort focused on the heater

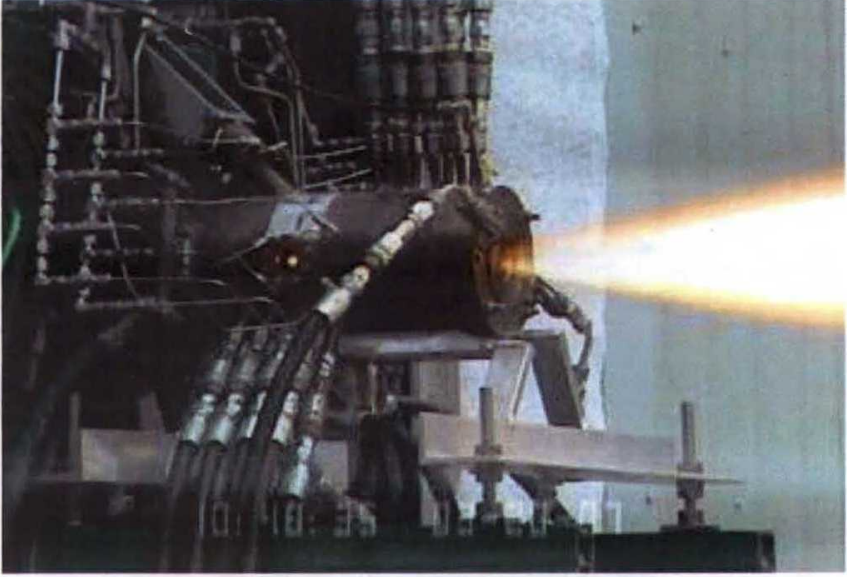

Figure 13. Long duration hot hydrogen testing image.

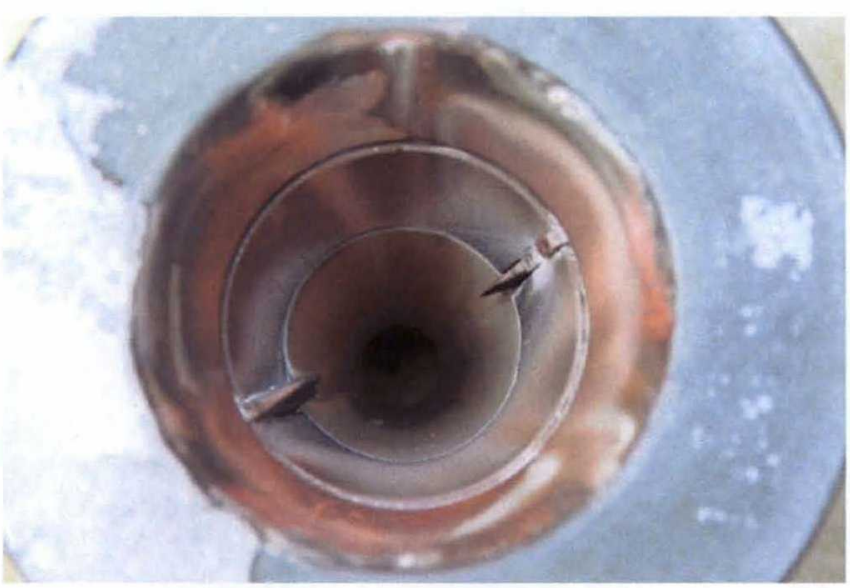

Figure 14. Post-test photograph of synthetic diamond wafer specimen. element and related components. At this point, it appears that we will have to revert to a design approach in which the heater element is suspended by its two electrical leads, the details of which must be resolved through future work.

\section{E. DU Filtration System}

Ultimately, meaningful non-nuclear fuels development testing would require utilization of specimens containing Depleted Uranium (DU). Because the specific activity of DU (less than $0.7 \%{ }^{235} \mathrm{U}$ ) is about 400 nanocuries per gram, however, it is classified as a low level transuranic waste and therefore subject to tight controls on handling and disposal. These controls are set by the US Nuclear Regulatory Commission (NRC) under Federal statutory authority established by the Atomic Energy Act, and a source material specific license from the NRC is required to receive, possess, use, transfer, and deliver DU. Because of these constraints on emission, it was necessary to design a suitable DU dilution/scrubber system to trap any DU that may escape from the material specimen and enter the exhaust stream of the NTR environments simulator.

A preliminary design for this dilution/scrubber system is illustrated in Fig. 15. The system first mixes the contaminated hot hydrogen exhaust with ambient temperature $\mathrm{GN}_{2}$ in order to reduce the gas temperature below 500 ${ }^{\circ} \mathrm{F}$, which is the temperature limit of the glass fiber HEPA filters being used to scrub the effluents. The amount of

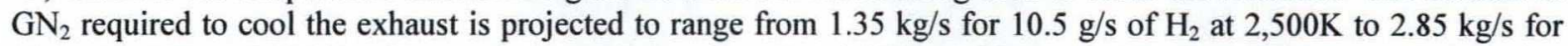
$10.5 \mathrm{~g} / \mathrm{s}$ of $\mathrm{H}_{2}$ at $3,500 \mathrm{~K}$. The preliminary design uses four $24^{\prime \prime} \mathrm{H} \times 24^{\prime \prime} \mathrm{W} \times 11.5^{\prime \prime} \mathrm{D}$ filters with a rated collection efficiency of $99.97 \%$ for $0.3 \square \mathrm{m}$ particles. The filters will be contained in a vender-supplied housing attached to 

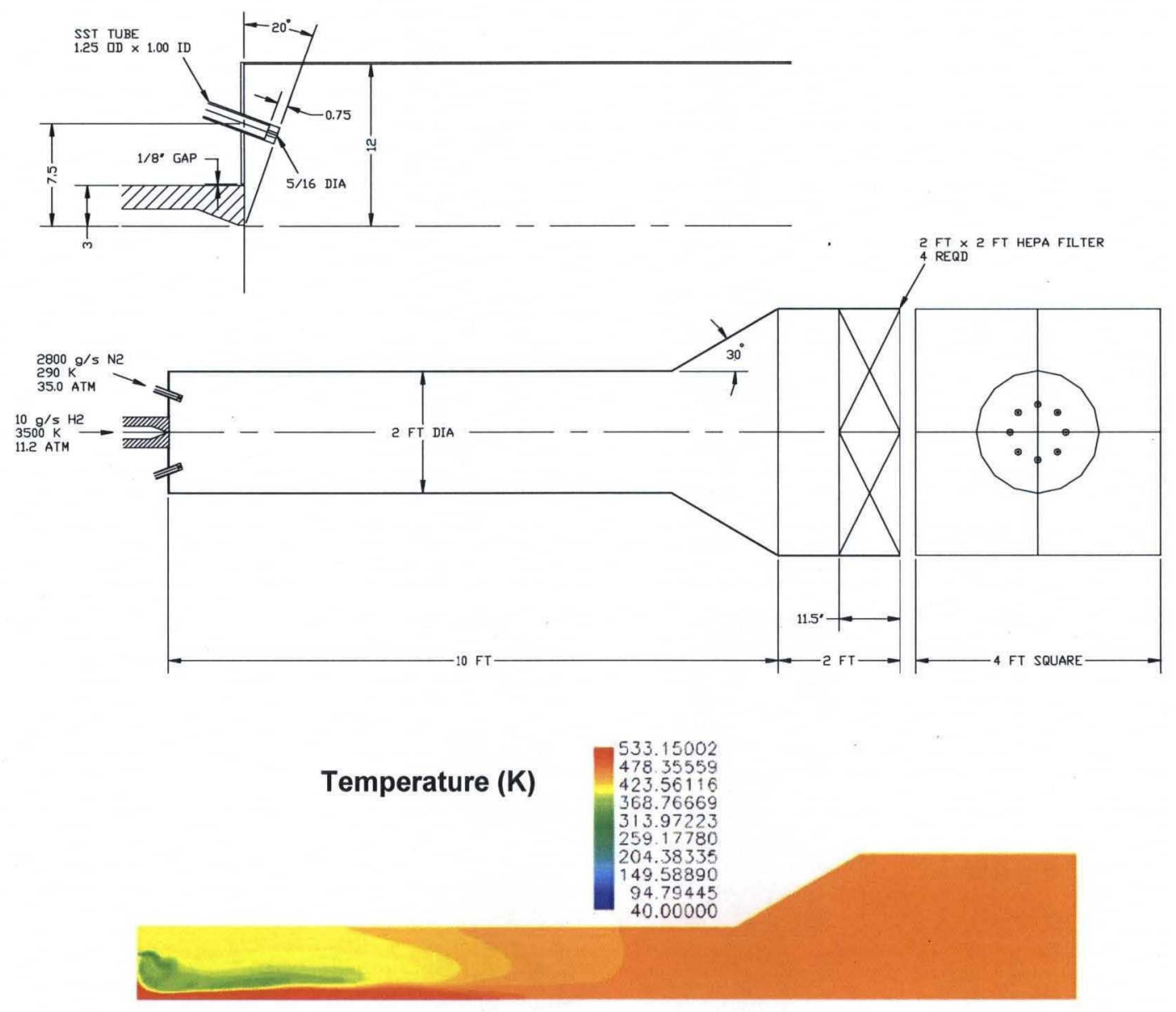

Figure 15. Preliminary design layout for dilution/scrubber system using industrial grade HEPA filters and projected flow-path temperature distribution from 3D CFD simulation.

custom fabricated ductwork. Three dimensional CFD simulations of the dilution/mixing process were performed to evaluate design performance, and the predicted flow-path temperature distribution is shown in Fig. 15. These results confirm that the flow is well mixed and below temperature limits when it reaches the filter housing inlet.

\section{Conclusion}

A preliminary design study was completed for extending the functionality of an existing non-nuclear NTR reactor environments simulator to support a critical examination of thermal hydraulic effects and hydrogen induced corrosion in fuel element passages. The existing baseline simulator, which uses a high-power wall-stabilized constricted arc-heater to generate hot hydrogen flow, was designed for the limited purpose of exposing small cylindrical material specimens in a modular water-cooled copper test fixture attachment. To achieve the desired functionality enhancement, a modified configuration was conceived whereby a short tubular material specimen, representing a fuel element passage, is surrounded by a backside resistive heater element and mounted within a selfcontained module that inserts directly into the baseline test fixture assembly. A detailed design layout was developed and comprehensive multi-physics thermal-fluid simulations were performed, including a conjugate heat transfer model with radiative transport. The results of these analyses confirmed that basic performance and functionality goals could be achieved with the proposed design configuration. It also demonstrated effective backside ohmic heating of the tubular specimen, development of an inward directed temperature gradient within the tube wall, and subsequent reheating of the hydrogen in the simulated passage. We conclude, therefore, that the 
resulting design configuration is capable of faithfully replicating thermal hydraulic characteristics in fuel element passages.

Material selection for the resistive curved slat heater element was a key technical issue, and several alternatives were considered. For instance, the viability of high density graphite coated with transition metal carbides was initially examined since the application required continuous operation above $2,300 \mathrm{~K}$. Several graphite sample specimens (3.5-inch long cylinders) were coated with $\mathrm{NbC}$ using direct and reaction EB-PVD and exposed to hot hydrogen in a high-temperature furnace. These coating trials generated mixed results, however, and were generally prone to suffer premature spallation, mainly due to thermal shock induced from limitations imposed by the testing configuration. Ultimately, the coated graphite approach was abandoned and rejected in favor of a tungsten curved slat design despite fabrication issues for a single piece element with tight tolerances and integral power leads. Additional testing was undertaken to examine the hot hydrogen compatibility of synthetic diamond wafers, which was proposed as an electrical insulator for directly supporting the heater element on the specimen. The material proved highly unsatisfactory, however, since it was found to rapidly ablate away in the hot hydrogen environment. This result revealed a significant flaw in the original configuration and reopened a knotty design issue related to structural support of the heater element. It is suggested that this problem could be resolved by suspending the heater element by its two electrical leads, but the details of this solution have been left to future work. Because practical utilization of the proposed non-nuclear reactor core simulator would ultimately require the testing of specimens containing depleted uranium, the free emission of which is barred, a preliminary design was also developed for a dilution/scrubber system based on industrial grade HEPA filters. Detailed 3D simulations confirmed that the resulting design would provide satisfactory dilution and mixing of the flow and reduce the gas temperature below the maximum upper limit of the filters.

The results of this preliminary design study, while somewhat incomplete, go a long way toward defining an affordable and workable design solution for a non-nuclear simulator that can satisfactorily replicate the thermal hydraulic conditions in an NTR reactor core fuel element passage. Perhaps the most significant utilization for an apparatus of this sort would be the generation of detailed quantitative information for guiding and validating physics-based modeling efforts for hydrogen induced corrosion. This non-nuclear approach could serve a highly valuable and practical utilitarian role in future high-temperature fuels development efforts with enormous cost savings and safety advantages, and is deserving of serious consideration for inclusion in any future NTR technology development program.

\section{Acknowledgments}

Funding for this work was derived from the NASA MSFC IR\&D program, Focus Area Project 2005-80 entitled "Hot Hydrogen Materials and Component Development," and, in part, from funds associated with FY2006 Congressionally Directed Item Reference No. 53 for Advanced Propulsion Materials Research supporting the development of future space exploration capabilities.

\section{References}

[1] D. R. Koenig, "Experiences Gained from the Space Nuclear Rocket Program (ROVER)," Los Alamos National Laboratory, Report LA-10062-H, May 1986.

[2] F. P. Durham, "Nuclear Engine Definition Study Preliminary Report," Los Alamos National Laboratory, Report LA-5044-MS, Vols. I - III, September 1972.

[3] J. D. Balcomb, "Nuclear Rocket Reference Data Summary," Los Alamos National Laboratory, Report LA-5057MS, October 1972.

[4] F. P. Durham, "The Nuclear Rocket Program at Los Alamos," American Institute of Aeronautics and Astronautics Paper AIAA-1969-556, June 1969.

[5] W. H. Robbins and H. B. Finger, "Historical Perspective of the NERVA Nuclear Rocket Engine Technology Program," NASA CR-187154, July 1991.

[6] J. R. Wetch, A. Ya. Goldin, A. A. Koroteev, A. D. Konopatov, V. A. Pavshook, N. N. Ponomarev-Stepnoy, and V. F. Semyonov, "Development of Nuclear Rocket Engines in the USSR," American Institute of Aeronautics and Astronautics Paper AIAA-1991-3648, September 1991. 
[7] J. M. Taub, "A Review of Fuel Element Development for Nuclear Rocket Engines," Los Alamos Scientific Laboratory, Report LA-5931, June 1975.

[8] L. L. Lyon, "Performance of (U,Zr)C-Graphite (Composite) and of (U,Zr)C (Carbide) Fuel Elements in the Nuclear Furnace 1 Test Reactor," Los Alamos Scientific Laboratory, Report LA-5398-MS, September 1973.

[9] E. K. Storms, D. Hanson, W. Kirk, and P. Goldman, "Effect of Fuel Geometry on the Life-Time Temperature Performance of Advanced Nuclear Propulsion Reactors," American Institute of Aeronautics and Astronautics Paper AIAA-1991-3454, September 1991.

[10] D. G. Pelaccio, M. S. El-Genk, and D. P. Butt, "Hydrogen Corrosion Considerations of Carbide Fuels for Nuclear Thermal Propulsion Applications," AIAA Journal of Propulsion and Power, Vol. 11, No. 6, 1995, pp. 1338-1348.

[11] M. S. El-Genk and D. G. Pelaccio (eds.), Proceedings of the Workshop on Hydrogen Corrosion in Nuclear Thermal Propulsion Reactors, 10th Symposium on Space Nuclear Power and Propulsion, Albuquerque, NM, 1994.

[12] R. J. Litchford, J. P. Foote, R. Hickman, C Dobson, and S. Clifton, "Long Duration Hot Hydrogen Exposure of Nuclear Thermal Rocket Materials," American Institute of Aeronautics and Astronautics Paper AIAA-2007-5625, July 2007. 